\title{
Multiparticle entanglement under asymptotic positive-partial-transpose-preserving operations
}

\author{
S. Ishizaka ${ }^{1,2}$ and M. B. Plenio ${ }^{3,4}$ \\ ${ }^{1}$ Fundamental and Environmental Research Laboratories, NEC Corporation, 34 Miyukigaoka, Tsukuba, 305-8501, Japan \\ ${ }^{2}$ PRESTO, Japan Science and Technology Agency, 4-1-8 Honcho Kawaguchi, 332-0012, Japan \\ ${ }^{3}$ QOLS, Blackett Laboratory, Imperial College London, Prince Consort Road, London SW7 2BW, United Kingdom \\ ${ }^{4}$ Institute for Mathematical Sciences, Imperial College London, 53 Exhibition Road, London SW7 2BW, United Kingdom
}

(Received 26 May 2005; published 25 October 2005; corrected 28 October 2005)

\begin{abstract}
We demonstrate that even under positive-partial-transpose-preserving operations in an asymptotic setting Greenberger-Horne-Zeilinger (GHZ) and $W$ states are not reversibly interconvertible. We investigate the structure of minimal reversible entanglement generating set (MREGS) for tripartite states under positive-partialtranspose- (PPT-) preserving operations. We demonstrate that the set consisting of $W$ and Einstein-PodolskyRosen (EPR) states alone cannot be an MREGS. In this context we prove the surprising result that the relative entropy of entanglement can be strictly subadditive for certain pure tripartite states which is crucial to keep open the possibility that the set of GHZ state and EPR states together constitute an MREGS under PPTpreserving operations.
\end{abstract}

DOI: 10.1103/PhysRevA.72.042325

PACS number(s): 03.67.Mn, 05.70.-a

\section{INTRODUCTION}

Constraints and resources are intimately related in physics. If we impose a constraint on a physical setting, then certain tasks become impossible. A resource must be made available to overcome the restrictions imposed by the constraints. Such a resource may be manipulated and transformed under the constrained set of operations but it emerges as a fundamental law that the resource cannot be created employing only the constrained set of operations.

An example, motivated by communication scenarios, is to impose the constraint of local quantum operations and classical communication (LOCC) in quantum mechanics. The resources that are implied by this constraint are nonseparable states and in particular pure entangled states such as singlet states, neither of which can be created by LOCC alone when starting from product states. This setting gives rise to a theory of entanglement as a resource under LOCC operations.

Any theory of entanglement as a resource will generally aim to provide mathematical structures to treat three questions: namely, (1) the characterization of entanglement, (2) the manipulation of entanglement, and (3) the quantification of the entanglement resource under the given constraint [1-6]. Of particular interest concerning the characterization of entanglement is the question of how many inequivalent types of entanglement there are within such a theory. For the theory of entanglement under LOCC operations we find that in the limit of infinitely many identically prepared copies of bipartite pure states, reversible-state transformations can be achieved [7]. As entanglement cannot be created under LOCC it must remain constant and it is therefore reasonable to say that the entanglement in different pure bipartite states is equivalent; i.e., there is only one kind of pure bipartite entanglement. The situation is much less transparent in the multiparticle setting. It has been proven in the tripartite setting that, for example, Greenberger-Horne-Zeilinger (GHZ) states

$$
|\mathrm{GHZ}\rangle=\frac{|000\rangle+|111\rangle}{\sqrt{2}}
$$

and Einstein-Podolsky-Rosen (EPR) states

$$
|\mathrm{EPR}\rangle=\frac{|01\rangle-|10\rangle}{\sqrt{2}}
$$

cannot be reversibly interconverted even in the asymptotic limit [8]. The question whether all other tripartite pure states can be created reversibly from GHZ and EPR states has also been considered and answered negatively $[9,10]$. The existence and structure of the smallest set of pure tripartite states that allows for the reversible generation of all other tripartite pure states, a so-called minimal reversible entanglement generating set (MREGS) [11], is still undecided and it is possible that such a set must contain infinitely many elements.

A different setting for an entanglement theory is motivated by the concept of partial time reversal or partial transposition. For two qubits, density matrices that remain positive under partial transposition [denoted as positive partial transpose (PPT) states] are exactly the separable states. For higher dimensions this is not the case as there are PPT states that are inseparable [12]. The positivity under partial transposition motivates the definition of the set of positive-partial-transpose-preserving operations (PPT operations) which are defined as those operations that map all PPT states to PPT states [13]. In this setting, the implied resource is all those states that are not positive under partial transposition.

The theory of entanglement under PPT operations still possesses the property that in an asymptotic setting purestate transformations are reversible and that consequently there is only one type of pure-state entanglement. The additional power afforded by PPT operations as compared to LOCC operations becomes apparent both in the mixed-state and multiparty setting. In the mixed-state setting examples 
for reversible-state transformations have been discovered [14] and the possibility remains open that in this setting all entanglement reduces to only one type, in stark contrast to the LOCC setting where reversible mixed-entanglement transformations are known only in trivial cases $[15,16]$. In the nonasymptotic setting for the pure state it has been shown that both under PPT operations [14] and under LOCC operations supported by PPT-bound entanglement [17] state transformations become possible that are impossible under LOCC. Indeed, it has been shown in [18] that on the singlecopy level we can use trace-preserving completely positive PPT operations to transform, for example, a GHZ state into a $W$ state,

$$
|W\rangle=\frac{|001\rangle+|010\rangle+|100\rangle}{\sqrt{3}},
$$

with a maximal success probability of

$$
p=\frac{1}{4}\left[-2+(18-6 \sqrt{3})^{1 / 3}+(18+6 \sqrt{3})^{1 / 3}\right] \approx 0.75 \ldots .
$$

This is remarkable in particular because under LOCC this transformation has a strictly zero success probability [19]. The surprisingly large success probability and the proven existence of some asymptotically reversible-state transformations under PPT operations in the bipartite setting suggests that a theory of entanglement under PPT operations might have a simpler structure than that under the LOCC constraint. Motivated by this we will consider the MREGS problem under the more general setting of PPT-preserving operations. We will show that $\mathrm{GHZ}$ and $W$ states remain asymptotically inequivalent even under PPT operations. We then explore possible PPT MREGS and find that the set consisting of the GHZ and EPR states is a very promising example for an MREGS under PPT-preserving operations.

\section{ASYMPTOTIC IRREVERSIBILITY BETWEEN GHZ AND $W$ STATE}

To demonstrate the asymptotic irreversibility between GHZ and $W$ states under PPT operations we will prove that there is one entanglement measure under which GHZ is strictly more entangled than the $W$ state and another entanglement measure where this relation is reversed. Such an example then provides a contradiction to the invariance of entanglement measures under reversible-state transformations. As we are considering an asymptotic setting, there are two constraints to be observed in the choice of entanglement measures. First, we have to employ the appropriate asymptotic versions $E^{\infty}$ of entanglement measures $E$ which are defined as

$$
E^{\infty}\left(\sigma_{A B C}\right)=\lim _{n \rightarrow \infty} \frac{E\left(\sigma_{A B C}^{\otimes n}\right)}{n} .
$$

Furthermore, we require that these asymptotic measures be continuous, as we must include in our considerations situations where the final state is reached asymptotically as a limit of progressively more similar states [14]. These requirements limit the choice of available entanglement measures quite severely.

The first two measures are based on the relative entropy of entanglement $[20,21]$ with respect to an appropriately defined set of states $\mathcal{T}$, which is given by

$$
E(\sigma)=\inf _{\rho \in \mathcal{T}} S(\sigma \| \rho)
$$

where $S(\sigma \| \rho)=\operatorname{Tr}\left\{\sigma \log _{2} \sigma-\sigma \log _{2} \rho\right\}$.

First, we consider the asymptotic two-party relative entropy of entanglement $E_{A: B C}(\sigma)$ across all the possible bipartite splits of the three parties. For pure states this measure can actually be computed analytically and equals the entropy of entanglement [7]-i.e., the entropy of the reduced density operator of one party. This measure is asymptotically continuous [22]. We then find

$$
E_{A: B C}\left(\sigma_{W}\right)=\log _{2} 3-\frac{2}{3}<1=E_{A: B C}\left(\sigma_{\mathrm{GHZ}}\right),
$$

where $\sigma_{W}=|W\rangle\langle W|$ and $\sigma_{\mathrm{GHZ}}=|\mathrm{GHZ}\rangle\langle\mathrm{GHZ}|$.

Second, we consider the tripartite relative entropy of entanglement with respect to states that are PPT for every bipartite split [24]:

$$
E_{A B C}\left(\sigma_{A B C}\right)=\inf _{\rho_{A B C} \in \text { tri-ppt }} S\left(\sigma_{A B C} \| \rho_{A B C}\right)
$$

Generally, the asymptotic relative entropy of entanglement is very difficult to compute except in situations with high symmetries [23], but useful lower bounds exist. Indeed, theorem 1 in [24] states that

$$
E_{A B C}^{\infty}\left(\sigma_{A B C}\right) \geqslant \max _{i=(A B),(A C),(B C)}\left\{E_{i}^{\infty}\left(\sigma_{i}\right)+S\left(\sigma_{i}\right)\right\},
$$

where $\sigma_{A B}=\operatorname{Tr}_{C} \sigma_{A B C}$ and $E_{i}$ is the relative entropy of entanglement for bipartite states. We then find

$$
E_{A B C}^{\infty}\left(\sigma_{\mathrm{GHZ}}\right)=1
$$

The final step of the proof consists in the verification of $E_{A B C}^{\infty}\left(\sigma_{W}\right)>1$. It is known $[22,27]$ that $E_{A B C}\left(\sigma_{W}\right)=\log _{2} 9 / 4$ but this is not sufficient as $E_{A B C}$ can be strictly subadditive for arbitrary pure tripartite states as we demonstrate in [23]. ${ }^{1}$ Indeed.

Lemma. The relative entropy of entanglement $E_{A B C}$ for the pure state (10) is strictly subadditive.

Proof. For the spin-0 state for a tripartite state of threelevel systems,

\footnotetext{
${ }^{1}$ After completion of this work we found that $\operatorname{Tr}_{A} \sigma_{W}$ satisfies Rains' additivity condition [29], and therefore the relative entropy of entanglement is weakly additive for $\operatorname{Tr}_{A} \sigma_{W}$. Using this and (8) it is found that $E_{A B C}^{\infty}\left(\sigma_{W}\right)=E_{A B C}\left(\sigma_{W}\right)=\log _{2} 9 / 4$ and hence the tripartite relative entropy of entanglement is weakly additive for the $W$ state.
} 


$$
|A\rangle=\frac{1}{\sqrt{6}} \sum_{i=1}^{3} \epsilon_{i j k}|i\rangle|j\rangle|k\rangle,
$$

where $\epsilon_{i j k}$ is the totally antisymmetric tensor, we find $E_{A B C}(|A\rangle\langle A|)=\log _{2} 6$, both with respect to LOCC states and with respect to PPT states. To demonstrate strict subadditivity of the tripartite relative entropy of entanglement we now consider the relative entropy of entanglement for two copies of $|A\rangle^{\otimes 2}$ given in Eq. (10). To this end we will provide a guess for a closest PPT state. We define the product state $|m\rangle=\left|m_{A}\right\rangle\left|m_{B}\right\rangle\left|m_{C}\right\rangle \quad$ with $\quad\left|m_{A}\right\rangle=\left|m_{B}\right\rangle=\left|m_{C}\right\rangle=(|00\rangle+|11\rangle$ $+|22\rangle) / \sqrt{3}$. Now we employ the invariance of $|A\rangle\langle A|$ under the application of $U \otimes U \otimes U$-i.e., twirling. Twirling the state $|m\rangle\langle m|$ leaves its overlap with $(|A\rangle\langle A|)^{\otimes 2}$ invariant so that we then find that the resulting state $\rho$ is of the form $\rho=F(|A\rangle\langle A|)^{\otimes 2}+\rho_{\perp}$ where $F=1 / 27$ and $\rho_{\perp}$ is orthogonal on $(|A\rangle\langle A|)^{\otimes 2}$. This state is certainly separable and therefore PPT. As a consequence we find

$$
\begin{aligned}
E_{A B C}\left((|A\rangle\langle A|)^{\otimes 2}\right) & \leqslant S\left((|A\rangle\langle A|)^{\otimes 2} \| \rho\right)=-\operatorname{tr}(|A\rangle\langle A|)^{\otimes 2} \log _{2} \rho \\
& =\log _{2} 27<2 \log _{2} 6=2 E_{A B C}(|A\rangle\langle A|) .
\end{aligned}
$$

This completes the proof of the lemma.

Straightforward additive lower bounds on the bipartite relative entropy of entanglement [25] such as

$$
E_{A B}\left(\sigma_{A B}\right) \geqslant \max \left\{S\left(\sigma_{A}\right), S\left(\sigma_{B}\right)\right\}-S\left(\sigma_{A B}\right)
$$

are not sufficient either to prove $E_{A B C}^{\infty}\left(\rho_{W}\right)>1$. We can, however, employ the distillable entanglement to lower bound the bipartite relative entropy of entanglement for the state $\operatorname{Tr}_{A} \rho_{W}$. In Sec. III B 2 of [26] a specific distillation employing two-way communication has been presented which, for the state $\operatorname{Tr}_{A} \rho_{W}$, yields an asymptotic gain of $D\left(\operatorname{Tr}_{A} \rho_{W}\right)=1 / 9$ of an EPR pair per copy. While the efficiency of this procedure can be increased [27] it is sufficient for our present purpose as it provides a lower bound on the asymptotic relative entropy of entanglement. With Eqs. (8) and (9) we then find

$$
E_{A B C}^{\infty}\left(\sigma_{W}\right) \geqslant \frac{1}{9}-\frac{1}{3} \log _{2} \frac{1}{3}-\frac{2}{3} \log _{2} \frac{2}{3}>1=E_{A B C}^{\infty}\left(\sigma_{\mathrm{GHZ}}\right) .
$$

As a consequence, Eqs. (6) and (11) and the continuity of the measures that we have employed demonstrate that it is impossible to satisfy both

$$
\begin{aligned}
& E_{A B C}^{\infty}\left(\sigma_{\mathrm{GHZ}}^{\otimes n_{\mathrm{GHZ}}}\right)=E_{A B C}^{\infty}\left(\sigma_{W}^{\otimes n_{W}}\right), \\
& E_{A: B C}^{\infty}\left(\sigma_{\mathrm{GHZ}}^{\otimes n_{\mathrm{GHZ}}}\right)=E_{A: B C}^{\infty}\left(\sigma_{W}^{\otimes n_{W}}\right)
\end{aligned}
$$

for any choice of $n_{W}$ and $n_{\mathrm{GHz}}$. Consequently, the asymptotic reversible transformation between GHZ and $W$ states is impossible even under PPT operations.

\section{EXPLORATION OF PPT MREGS}

The result proven above implies that neither the set $\{\mathrm{GHZ}\}$ nor $\{W\}$ forms a PPT MREGS. In the following we will explore whether the addition of a set of EPR states $\left\{\mathrm{EPR}_{A B}, \mathrm{EPR}_{A C}, \mathrm{EPR}_{B C}\right\}$ can suffice to create an PPT MREGS.

Let us begin by considering the set $\left\{W, \mathrm{EPR}_{A B}, \mathrm{EPR}_{A C}, \mathrm{EPR}_{B C}\right\}$ and explore whether it is possible to obtain a GHZ state reversibly from this set. Let us denote

$$
\sigma_{\mathrm{WEPR}}=\sigma_{W}^{\otimes n_{W}} \otimes \mathrm{EPR}_{A B}^{\otimes n_{A B}} \otimes \mathrm{EPR}_{A C}^{\otimes n_{A C}} \otimes \mathrm{EPR}_{B C}^{\otimes n_{B C}},
$$

with positive $n_{W}, n_{A B}, n_{A C}, n_{B C}$. Reversibility then requires that

$$
\begin{aligned}
& E_{A B C}\left(\sigma_{\mathrm{WEPR}}\right)=E_{A B C}\left(\sigma_{\mathrm{GHZ}}^{\otimes n_{\mathrm{GHZ}}}\right), \\
& E_{A: B C}\left(\sigma_{\mathrm{WEPR}}\right)=E_{A: B C}\left(\sigma_{\mathrm{GHZ}}^{\otimes n_{\mathrm{GHZ}}}\right), \\
& E_{B: A C}\left(\sigma_{\mathrm{WEPR}}\right)=E_{B: A C}\left(\sigma_{\mathrm{GHZ}}^{\otimes n_{\mathrm{GHZ}}}\right), \\
& E_{C: A B}\left(\sigma_{\mathrm{WEPR}}\right)=E_{C: A B}\left(\sigma_{\mathrm{GHZ}}^{\otimes n_{\mathrm{GHZ}}}\right)
\end{aligned}
$$

be satisfied. As a consequence of the permutation symmetry of the $W$ and GHZ states we find that $n_{A B}=n_{A C}=n_{B C}=n$ and in the asymptotic limit Eqs. (13)-(15) all lead to

$$
n_{W} H\left(\frac{1}{3}\right)+2 n=n_{\mathrm{GHZ}} \text {. }
$$

Due to the lack of general additivity of $E_{A B C}$, we need to employ in Eq. (12) the bound of Eq. (8) as well as the additivity of $E_{2}^{\infty}(\rho \otimes \sigma)=E_{2}^{\infty}(\rho)+E_{2}^{\infty}(\sigma)$ for a pure as well as separable $\sigma$ [13]. Then we find

$$
n_{W}\left[E_{2}^{\infty}\left(\operatorname{Tr}_{A} W\right)+H\left(\frac{1}{3}\right)\right]+3 n \leqslant n_{\mathrm{GHZ}} .
$$

Employing again that $E_{2}^{\infty}\left(\operatorname{Tr}_{A} W\right) \geqslant \frac{1}{9}$ we find that Eqs. (16) and (17) can only be satisfied when $n<0$ which is a contradiction.

The above result suggests that a more promising choice for the PPT MREGS is $\left\{\mathrm{GHZ}, \mathrm{EPR}_{A B}, \mathrm{EPR}_{A C}, \mathrm{EPR}_{B C}\right\}$. Let us now consider whether it is possible to obtain the $W$-state reversibly from this set. Let us denote

$$
\sigma_{G}=\sigma_{\mathrm{GHZ}}^{\otimes n_{\mathrm{GHZ}}} \otimes \mathrm{EPR}_{A B}^{\otimes n_{A B}} \otimes \mathrm{EPR}_{A C}^{\otimes n_{A C}} \otimes \mathrm{EPR}_{B C}^{\otimes n_{B C}},
$$

with positive $n_{A B}, n_{A C}, n_{B C}$, and $n_{\mathrm{GHZ}}$. Reversibility then implies

$$
\begin{aligned}
& E_{A B C}\left(\sigma_{G}\right)=E_{A B C}\left(\sigma_{W}^{\otimes n_{W}}\right), \\
& E_{A: B C}\left(\sigma_{G}\right)=E_{A: B C}\left(\sigma_{W}^{\otimes n}\right), \\
& E_{B: A C}\left(\sigma_{G}\right)=E_{B: A C}\left(\sigma_{W}^{\otimes n_{W}}\right), \\
& E_{C: A B}\left(\sigma_{G}\right)=E_{C: A B}\left(\sigma_{W}^{\otimes n_{W}}\right) .
\end{aligned}
$$

Again, Eqs. (19)-(21) imply $n_{A B}=n_{A C}=n_{B C}=n$. Due to the additivity of $E_{A B C}$ for the state $\sigma_{G}$, we find in the asymptotic limit the equations

$$
n_{\mathrm{GHZ}}+3 n=n_{W} E_{A B C}^{\infty}\left(\sigma_{W}\right),
$$




$$
n_{\mathrm{GHZ}}+2 n=n_{W} H\left(\frac{1}{3}\right) .
$$

These two equations are solved by

$$
\begin{gathered}
\frac{n}{n_{W}}=E_{A B C}^{\infty}\left(\sigma_{W}\right)-H\left(\frac{1}{3}\right)>0, \\
\frac{n_{\mathrm{GHZ}}}{n_{W}}=3 H\left(\frac{1}{3}\right)-2 E_{A B C}^{\infty}\left(\sigma_{W}\right)>0 .
\end{gathered}
$$

We are therefore unable to rule out that a $W$ state can be generated asymptotically reversibly from $\left\{\mathrm{GHZ}, \mathrm{EPR}_{A B}, \mathrm{EPR}_{A C}, \mathrm{EPR}_{B C}\right\}$. In general, using a set of constraints analogous to Eqs. (18)-(21), it is found that necessary constraints so that a tripartite state $|\psi\rangle$ can be generated reversibly from the set are

$$
\max \left\{E_{A: B C}(\psi), E_{B: A C}(\psi), E_{C: A B}(\psi)\right\} \leqslant E_{A B C}^{\infty}(\psi)
$$

and

$$
E_{A B C}^{\infty}(\psi) \leqslant \frac{E_{A: B C}(\psi)+E_{B: A C}(\psi)+E_{C: A B}(\psi)}{2} .
$$

The former constraint, Eq. (22), is satisfied for an arbitrary $|\psi\rangle$ because of the lower bound for $E_{A B C}^{\infty}(\psi)$ of Eq. (8). For the $W$ state, the latter constraint of Eq. (23) can be satisfied even if the relative entropy of entanglement is additive because

$$
E_{A B C}^{\infty}\left(\sigma_{W}\right) \leqslant E_{A B C}\left(\sigma_{W}\right)<\frac{3}{2} H\left(\frac{1}{3}\right) .
$$

For some tripartite pure states, however, the subadditivity of the relative entropy of entanglement plays a crucial role for the satisfaction of Eq. (23) as shown below.

The spin-0 state given by Eq. (10) exhibits strict subadditivity of the relative entropy of entanglement as had been shown in the lemma. From [24] we find a lower bound of $\log _{2} 5$ on the asymptotic relative entropy of entanglement with respect to PPT states. The constraint, Eq. (23), requires that $E_{A B C}^{\infty}(A) \leqslant \frac{1}{2} \log 27 \approx 1.02 \log 5$. While we do not know the asymptotic relative entropy of entanglement $E_{A B C}^{\infty}(A)$ precisely we can bound it from above by providing an upper bound on $E\left(A^{\otimes 2}\right)$ which in turn yields an upper bound on $E_{A B C}^{\infty}(A)$. We find that $E\left(A^{\otimes 2}\right) \leqslant \log 27$ which again shows that we cannot rule out that $\left\{\mathrm{GHZ}, \mathrm{EPR}_{A B}, \mathrm{EPR}_{A C}, \mathrm{EPR}_{B C}\right\}$ is an PPT MREGS.

\section{CONCLUSION}

In the single-copy setting for tripartite entanglement it can be shown that a single copy of a GHZ state can be converted to a $W$ state with a success probability of more than $75 \%$ employing trace-preserving PPT operations. Motivated by this we have investigated the manipulation of tripartite states with PPT-preserving operations in the asymptotic setting. The large success probability for the GHZ to $W$ transformation in the single-copy setting could have pointed to a possible asymptotic reversibility of these states or, at least, a simpler structure of MREGS under PPT-preserving operations. Despite this large success probability, however, we have been able to demonstrate that even in an asymptotic setting GHZ and $W$ states do not become reversibly interconvertible. We furthermore explored the minimal size of a MREGS under PPT-preserving operations. We have been able to prove that the set consisting of $W$ and EPR states alone cannot form an MREGS. The set of GHZ state and EPR states, however, constitutes a promising candidate for an MREGS under PPT-preserving operations. This result suggest that by comparison to the situation under LOCC operations the structure of MREGS under PPT-preserving transformations might be simplified.

\section{ACKNOWLEDGMENTS}

We are grateful to K. Audenaert for his help in finding the example provided in the proof of the lemma. We also acknowledge discussions with B. Groisman, N. Linden, S. Popescu, and A. Winter on the issues discussed here. This research was initiated during two visits to the ERATO project on "Quantum Computation and Information." This work is part of the QIP-IRC (www.qipirc.org) supported by EPSRC (Grant No. GR/S82176/0) as well as the EU (Grant No. IST-2001-38877), the Royal Society, and the Leverhulme Trust.
[1] M. B. Plenio and V. Vedral, Contemp. Phys. 39, 431 (1998).

[2] M. B. Plenio and V. Vitelli, Contemp. Phys. 42, 25 (2001).

[3] M. Horodecki, Asia-Pacific Build. Constr. Manage. J. 1, 3 (2001).

[4] P. Horodecki and R. Horodecki, Quantum Inf. Comput. 1, 45 (2001).

[5] J. Eisert and M. B. Plenio, Quantum Inf. Comput. 1, 479 (2003).

[6] M. B. Plenio and S. Virmani, e-print quant-ph/0504163.

[7] C. H. Bennett, H. J. Bernstein, S. Popescu, and B. Schumacher, Phys. Rev. A 53, 2046 (1996).
[8] N. Linden, S. Popescu, B. Schumacher, and M. Westmoreland, e-print quant-ph/9912039.

[9] E. F. Galvão, M. B. Plenio, and S. Virmani, J. Phys. A 33, 8809 (2000).

[10] W. Dür, G. Vidal, and J. I. Cirac, Phys. Rev. A 62, 062314 (2000).

[11] C. H. Bennett, S. Popescu, D. Rohrlich, J. A. Smolin, and A. V. Thapliyal, e-print quant-ph/9908073.

[12] M. Horodecki, P. Horodecki, and R. Horodecki, Phys. Rev. Lett. 80, 5239 (1998).

[13] E. M. Rains, IEEE Trans. Inf. Theory 47, 2921 (2001).

[14] K. Audenaert, M. B. Plenio, and J. Eisert, Phys. Rev. Lett. 90, 
027901 (2003).

[15] G. Vidal and J. I. Cirac, Phys. Rev. Lett. 86, 5803 (2001).

[16] M. Horodecki, A. SenDe, and U. Sen, Phys. Rev. A 67, 062314 (2003).

[17] S. Ishizaka, Phys. Rev. Lett. 93, 190501 (2004).

[18] S. Ishizaka and M. B. Plenio, Phys. Rev. A 71, 052303 (2005).

[19] W. Dür, G. Vidal, and J. I. Cirac, Phys. Rev. A 62, 062314 (2000).

[20] V. Vedral, M. B. Plenio, M. A. Rippin, and P. L. Knight, Phys. Rev. Lett. 78, 2275 (1997); V. Vedral, M. B. Plenio, K. Jacobs, and P. L. Knight, Phys. Rev. A 56, 4452 (1997).

[21] V. Vedral and M. B. Plenio, Phys. Rev. A 57, 1619 (1998).

[22] M. J. Donald, and M. Horodecki, Phys. Lett. A 264, 257 (1999); M. J. Donald, M. Horodecki, and O. Rudolph, J. Math. Phys. 43, 4252 (2002).
[23] K. Audenaert, J. Eisert, E. Jané, M. B. Plenio, S. Virmani, and B. DeMoor, Phys. Rev. Lett. 87, 217902 (2001).

[24] M. B. Plenio and V. Vedral, J. Phys. A 34, 6997 (2001).

[25] M. B. Plenio, S. Virmani, and P. Papadopoulos, J. Phys. A 33, L193 (2000).

[26] C. H. Bennett, D. P. DiVincenzo, J. A. Smolin, and W. K. Wootters, Phys. Rev. A 54, 3824 (1996).

[27] We have been able to increase the yield to approximately 0.17 . The procedure is unlikely to be optimal and it is conceivable that one can achieve $E_{D}\left(\operatorname{tr}_{A} \rho_{W}\right)=\log _{2} 3-\frac{4}{3}$ which would guarantee the additivity of $E_{A B C}$ for the $W$ state.

[28] T.-C. Wei, M. Ericsson, P. M. Goldbart, and W. J. Munro, Quantum Inf. Comput. 4, 252 (2004).

[29] E. Rains, Phys. Rev. A 60, 179 (1999); 63, 019902(E) (2001). 